\title{
Anterior skull base surgery
}

\author{
Moni Abraham Kuriakose · Nirav P Trivedi · Vikram Kekatpure
}

Received: 20 December 2009

Accepted: 3 April 2010

(C) Indian Association of Surgical

Oncology 2010

Moni Abraham Kuriakose ( $₫)$.

Nirav P Trivedi · Vikram Kekatpure

Department of Head and Neck Oncology

Mazumdar Shaw Cancer Center

Narayana Health City

Bangalore 560 099, India

e-mail: makuriakose@gmail.com, mak12@nyu.edu

\begin{abstract}
The basic principle of anterior skull base surgery is to provide adequate exposure to enable three dimensional resection of skull base tumors. Negative surgical margins, which is within the control of surgeon, is the principle prognostic factor in anterior skull base tumors. Open skull base approaches is the standard of care for malignant anterior skull base tumors. Benign lesions may be resected by alternate minimally invasive approaches. Advances in anterior skull base surgery, in particular the facial translocation approaches allows wide exposure of the tumors with minimal retraction of the brain. The outcome of anterior skull base tumors have steadily increased over the years with disease free survival comparable to other malignant neoplasm of the head and neck region. This review described various surgical approaches and pertaining anatomy and pathology of anterior skull base tumors.
\end{abstract}

Keywords Anterior skull base surgery $\cdot$ Skull base surgery $\cdot$ Craniofacial resection $\cdot$ Skull base reconstruction

\section{Introduction}

Anterior skull base tumors are divers group of disease with different tumor biology, treatment approaches and clinical behavior. The proximity to vital structures prevents intensification for treatment either by surgery or radiotherapy, without unduly increasing morbidity. With improved understanding of skull base anatomy, tumor biology, surgical access and precision radiotherapy techniques, there has been steady improvement in the treatment outcome- both in terms of disease free survival and morbidity of treatment.

Surgery remains the important modality of treatment of anterior skull base tumors. Anterior skull base surgery has evolved greatly with better understanding of anatomy, pathology, imaging and surgical techniques. Advent of microvascular free flap reconstruction has made reconstruction of complex skull base defects possible and hence increased the scope of tumor ablation. But the goals of anterior skull base surgery have remained constant. These goals include:
1. Resection of tumor with three dimensional negative margins.

2. Preservation of important structures and their function.

3 Reconstruction of defect to maintain function and aesthesis.

Surgical approaches to the anterior skull base have been described as early as in 1954. ${ }^{1}$ Earlier reports suggested the likely hood of cure is better with surgery than other modalities of treatment, however with significant morbidity. ${ }^{2-5}$. Over the years, many authors have described various techniques to improve access for tumor resection and effective reconstruction. ${ }^{6,7}$ Endoscopic approaches have been recently introduced to further reduce morbidity of resection skull base resection. ${ }^{7}$ With all these advances, the results have improved significantly in recent past and the anterior skull base surgery has become a routine surgical procedures in many head and neck oncology services.

This review will focus on applied anatomy, pathology, and techniques of resection and reconstruction of anterior skull base tumors. 


\section{Applied anatomy}

The anterior skull base includes the posterior wall of frontal sinus, cribriform plate and the planum sphenoidale. Just below cribriform plate is ethmoid sinus which is separated from orbit by lamina papyracea. Orbital roof forms the lateral part of anterior skull base, ${ }^{8,9}$ Understanding relationship of tumor to vital structures are essential to preserve these structures and to minimize morbidity of treatment.

\section{Orbit preservation}

Anatomically, contents in orbit can be named chronologically from outside-in - orbital periosteum- peri-orbita, orbital fascia, orbital fat, rectus muscle and globe. Koorneef et al. ${ }^{72}$ has observed fine lamellae traversing the orbital fat and connect ocular muscles, thereby coordinating the globe movements. Entrapment or scarring of these lamellae can interfere with eye movements.

Lannetti et al. has identified three stages of orbital invasion: grade I, erosion or destruction of the osseous orbital wall; grade II, extraconal invasion of the periorbital fat grade III, invasion of the rectus muscle, optic nerve, ocular bulb, or the skin overlying the eyelid 10 . They propose that only grade III orbital invasion warrants orbital exenteration. Tiwari RM., highlighted the importance of peri-orbital fascia lining the orbital fat in preserving the orbit. ${ }^{11}$ Accordingly, tumor can be safely resected without exenteration if orbital fat is not involved. Even with the most accurate imaging combining thin section CT and MRI, frozen section confirmation is required at the time of surgery to determine whether tumor has infiltrated orbital fat.

\section{Internal carotid artery, Optic nerve and Opthalmic artery}

The ophthalmic artery arises from the internal carotid artery (ICA) superiorly or superomedially and then courses through the optic canal along the optic nerve. It typically runs on the lateral surface of the nerve at the anterior optic canal. Thus, dissection of the anterior optic chiasm and the medial optic canal is generally safe.

The optic nerve and ICA are usually covered by bone in the superolateral aspect of the sphenoid sinus, although in some cases the bone may be dehiscent. The optic nerve lies just superior to the ICA. Careful review of the preoperative MRI is essential to understand the anatomic relationships in a particular patient. Intraoperative navigation system is useful to guide resection near vital structures.

\section{Cribriform plate and dura}

The olfactory apparatus lies just above the cribriform plate, which itself lies just medial and usually slightly inferior to the ethmoid roof. Dura is much more tightly adherent at the cribriform plate than at the ethmoid roof. A thin-section $(1 \mathrm{~mm})$ CT scan with coronal reformatting is useful for assessing the bony integrity of the cribriform plate and ethmoid roof. This area can also be assessed with MRI, which better depicts dural and parenchymal extension of tumor and enable distinguishing tumor from post-obstructive changes within pananasal sinuses. If the olfactory apparatus is involved only minimally and unilaterally, one can consider preservation of the contralateral olfactory apparatus.

\section{Pathology}

Tumors that may require anterior skull base resection include malignant tumors of the nose and paranasal sinuses that extend superiorly through the cribriform plate, ethmoid roof, and planum sphenoidale. Biological behavior of different tumors are one most the most important prognostic factor in anterior skull base region. Overall outcome is significantly for different pathological type of tumors. Below are brief overviews of selected histologies. ${ }^{15-17}$

\section{Squamous cell carcinoma}

The most common malignancy of the paranasal sinuses is squamous cell carcinoma (SCC). SCC most commonly arises in the maxillary sinus, but extension posteriorly and supero-medially may make it necessary to undertake resection by skull base approaches to obtain resection uninvolved margins. Brain invasion and positive margins are two critical prognostic factors. Of which margin status is only under the control of surgeons. It is absolutely essential to obtain resection with negative margin. Surgery followed by radiotherapy is the standard of care for SCC.

\section{Adenocarcinoma}

Adenocarcinoma accounts for $10 \%$ to $15 \%$ of paranasal sinus malignancies in the United States and as many as $40 \%$ in Asia. Woodworking and the leather industry are etiologic factors. Surgery and postoperative irradiation yields local control and cure rates of $40 \%$ to $50 \%$. Chance of cervical metastasis is $10-15 \%$ and careful assessment of neck is warranted. ${ }^{18}$ Distant metastases are unusual.

\section{Adenoid cystic carcinoma}

These tumors account for $10 \%$ to $15 \%$ of paranasal sinus malignancies. Adenoid cystic carcinoma has a unique 
biological behavior showing strong tendency for perineural spread and local recurrence is as high as $33 \%$. ${ }^{19}$ Large number of patients also demonstrates distant metastasis mainly to lungs. All these patients with local or distant disease still live for long duration. As a rough guide, at 10 year follow up, about $33 \%$ will be alive disease free, $33 \%$ alive with disease and about $33 \%$ dead of disease.

\section{Esthesioneuroblastoma (olfactory neuroblastoma)}

Esthesioneuroblastomas are rare tumors that arise from the olfactory epithelium and hence are typically seen at the level of the cribriform plate. ${ }^{20-22}$ Dural involvement at the level of the olfactory groove is usually present, even when preoperative MRI indicates no apparent involvement superior to the cribriform plate. This involvement makes an anterior skull base approach necessary for management of almost all esthesioneuroblastomas. Extensive involvement of dura and even brain invasion may be present initially or may occur in the context of recurrent disease. Nodal or distant metastases are rare at presentation.

\section{Mucosal melanoma}

Melanomas of the nasal cavity and paranasal sinuses are associated with a poor prognosis because of high rates of local recurrence and of distant metastases. ${ }^{23,24}$ Surgery and postoperative irradiation are commonly employed. Protocols involving interferon and chemotherapy are available.

\section{Sinonasal undi erentiated carcinoma}

Sinonasal undi erentiated carcinoma is a rare, highly aggressive malignancy that commonly presents with extensive local involvement and often involves the orbit and skull base 25,26 . Neck metastases are seen in $20 \%$ of patients at presentation. There are very high rate of distant metastasis. It is important to differentiate it from esthesioneuroblastoma and lymphoma and immunohistochemistry is essential in establishing the diagnosis.

\section{Chordoma}

Approximately one third of chordomas arise in the clivus and extend toward the craniocervical junction. Another $15 \%$ occur in cervical vertebrae, and the remainder are sacrococcygeal in origin. The typical age at presentation is 35 to 50 years. Typical symptoms are headache and diplopia secondary to cranial nerve VI paresis and sensory $\mathrm{V}$ deficits at cranial nerve $\mathrm{V}$ region are common. Chordomas are often large at presentation and may abut or encase the cavernous segment of the ICA or the vertebrobasilar system. Treatment is complete resection. About $90 \%$ of tumors at the skull base fall into this favorable category. Larger or recurrent tumors do less well, with a 5 -year cure rate of about $33 \%$.

\section{Lymphoma}

Lymphomas represent about $10 \%$ of nonepithelial malignancies of the paranasal sinuses. Diffuse large cell B-cell lymphoma is most common, usually presenting as stage 1E. With current multimodality therapy, two thirds of these patients are cured. NK/T-cell lymphomas, usually also Epstein-Barr virus-positive, represent about a third of lymphomas when predominantly nasal involvement is seen. IHC is essential in establishing diagnosis.

\section{Angiofibroma}

Juvenile angiofibromas are locally invasive, highly vascular benign tumors that occur in male adolescents. Common symptoms are epistaxis and nasal obstruction. Originating at the junction of the posterolateral nasal wall and sphenoid rostrum near the sphenopalatine foramen, they frequently extend laterally into the pterygopalatine fossa, superoposteriorly into the sphenoid sinus, or superiorly to involve the skull base. Anterior extension results in nasal obstruction. The appearance on MRI of a tumor with macroscopic flow voids at this location in an adolescent male is rarely confused with another diagnosis.

\section{Limits of resectability}

Absolute contraindication for resection of anterior skull base tumors are bilateral orbit involvement and internal carotid artery involvement. Relative contraindication for excision are involvement of brain and cavernous sinus involvement. But studies have suggested that even these tumors can be resected safely and final outcome depends on tumor biology. ${ }^{12-14}$

\section{Surgical approaches}

The intimate relationship of the skull base to brain and facial structures requires displacement of either or both of these structures to gain access to the skull base. Effects of such surgical displacement have variable consequences. While facial swelling is self-limiting, edema of the brain has serious long-term deleterious effects. So in general it is preferable to displace the facial viscerocranium than the neurocranium. This prompted the development of facial translocation approaches to the skull base. 
Various trans-facial and trans-cranial approaches have been developed to obtain three dimensional exposure, resection with negative margins and lower morbidity of the procedure. Choice of approach depends on extent of involvement of nose or paranasal sinus, the angle of approach so as to minimize brain retraction, the extent of dural or intradural extension and the need for access to difficult and critical sites such as the optic chiasm, internal carotid artery, nasopharynx or craniocervical junction. Endoscopic approach to resect these tumors is beyond scope of this article, which will be described elsewhere in this issue of the journal. This review will focus on various open trans-facial and trans-cranial approaches and various reconstructive options.

\section{Trans-cranial approach}

The anterior skull base has classically been approached through a coronal incision and bifrontal craniotomy with retraction of the frontal lobe. Standard coronal incision starts from the pre-auricular region of one ear to other ear. The flap is elevated at subpericranial plane, so as to use Galleo-pericranial flap based on supra-orbital and supra trochlear vessels anteriorly. This flap may be best to harvest at the time of repair of the dural repair. Bifrontal craniotomy is carried out and bone segment is removed. Opening of lumbar drain decreases the tension and makes it easy for retraction of frontal lobe. Dura is carefully separated from cribriform plate. Part of dura, and frontal pole can be resected depending upon extent of tumor. Both side olfactory tract can be identified and divided. (Fig. 1).

This approach gives excellent exposure for resection intracranial part of tumor. The disadvantage is that significant brain retraction is required to achieve satisfactory

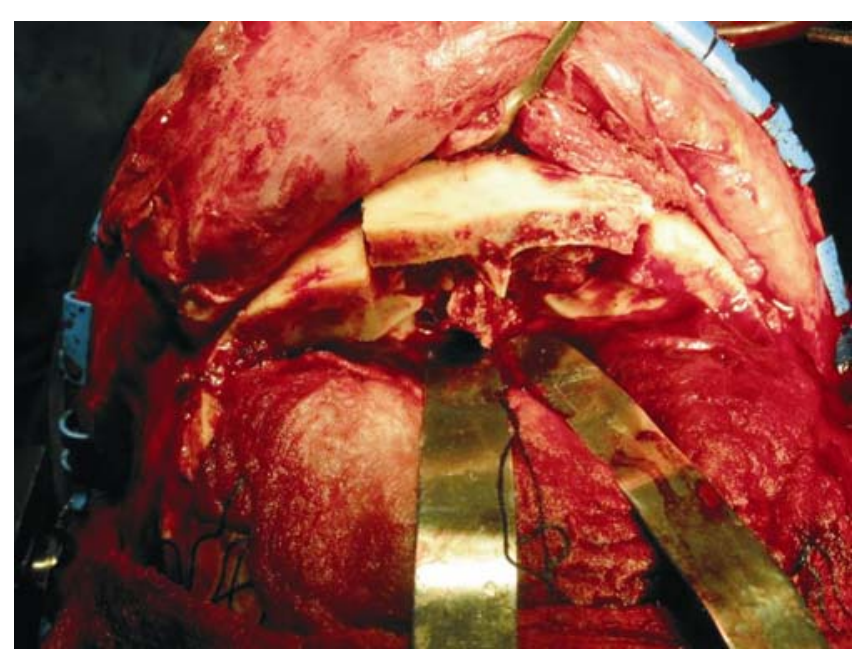

Fig. 1 Trans-cranial approach with bifrontal craniotomy and frontal lobe retraction to access anterior skull base exposure. Currently, this approach is used only if tumor has significant intracranial extension or patient has very small frontal sinus which makes subcranial approach difficult. This approach has been used in combination with endoscopic approach to resect few benign and small volume malignant tumors. ${ }^{28-34}$

\section{Subcranial approach}

Raveh et al. ${ }^{35}$ have popularized the subcranial approach through a bicoronal incision that was initially described for trauma but has been extended to tumor resection. The major advantage of this low craniotomy is minimization of brain retraction (Fig. 2). Two type of classical subcranial approaches are described. In type A, anterior wall of frontal sinus is removed along with the nasal bone. The posterior wall is then removed and the frontal sinus cranialized, as deemed required for tumor resection. In type B approach- a full thickness craniotomy of supra orbital rim and both walls of the frontal sinus are involved in the
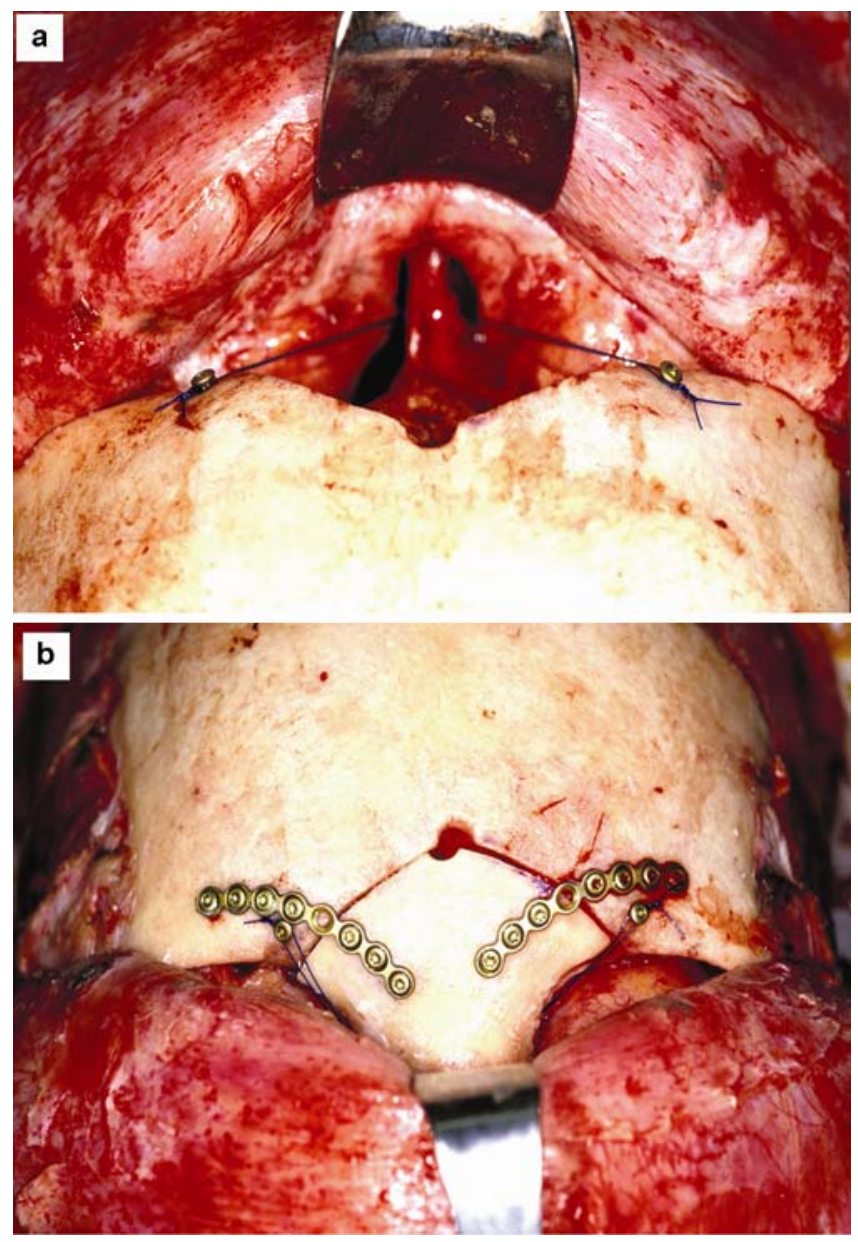

Fig. 2a Subcranial Approach- exposure and fixation of medial canthus. b Subcranial approach- repositioning of anterior frontal sinus wall 
bone flap. The posterior frontal sinus wall may be removed later to cranialize the sinus. This approach gives excellent exposure for tumor resection with minimal brain retraction. Extensive dural resection and reconstruction may not be possible with this approach. Another potential disadvantage of the subcranial approach when it is used in resection of malignant tumors is osteomyelitis or osteoradionecrosis of the disconnected and replaced bone that includes the medial orbital rim, glabella, and part of the nasal bone 17 . This may be avoided by wrapping the bone flap with galleopericranial flap. Fixation of medial canthal ligament to nasal bone at end of procedure is important step to prevent telecanthus.

\section{Facial translocation approach}

Facial translocation is based on the observation that facial functional and aesthetic units can be disassembled to gain access to the skull base and can be repositioned without causing significant functional or aesthetic impairment. Development of face is through the fusion of embryological naso-frontal, maxillary and mandibular processes. The fusion of these processes takes place at the midline or the paramedian region. Each of these processes has distinct neurovascular territory. Therefore separation of the face respecting these embryologic principle cause minimal disruption to the vital structures.

This concept is well established for over 50 years in the correction of craniofacial deformities. For example to correct hypertelorism caused by lateral displacement of ophthalmic units it is possible to osteotomize around the orbits and bring the displaced orbits to normal anatomic relationship. Similarly there are several well established orthognathic surgical procedures to correct abnormal relationship between facial skeleton and that to the skull base.

Facial translocation approach is a direct application of this principle in skull base surgery. This technique was introduced by Ivo Janecka in 1990. Disassembly of facial skeleton allows excellent exposure of the skull base tumor and minimizes the need for brain retraction. Retraction of the brain is one of the main cause of morbidity and mortality of skull base resection. It also allows rapid access to skull base and provides generous surgical working space. Moreover facial translocation allows threedimensional resection of tumor and provides the opportunity to functional reconstruction of the defect.

Classification: Depending on the extent skull base exposure and resection, facial bone translocation procedure can be classified into five groups.

- Zygomatico-maxillary translocation (Standard facial translocation)

- Tras-zygomatic
- Medial maxillotomy

- Extended facial translocation

- Mandibulo-maxillary translocation

- Bilateral facial translocation

The surgery is designed to translocate composite facial units with its neurovascular supply. In effect facial units are reassembled in modules. Therefore it would be possible to combine any of the above procedures in a modular fashion. This ability for modular disassembly permits surgeon to tailor the procedure depending on the extent skull base exposure. Depending on the type of approach used it allows exposure of the entire anterior and middle cranial fossa except for the peterous temporal bone.

\section{Surgical technique}

Incision: A standard Webber-Ferguson incision is employed for this procedure, with certain modification to improve functional and aesthetic results. The vertical part of the incision may be extended into the anterior nares and then curved along the alar cartilage. It is further carried superiorly midway between the midline of the nose and the nasolabial fold up to the medial canthus level. At that point it is carried laterally to bisect the medial canthus and then through the inferior fornix of the lower eyelid to the lateral canthus, as in a trans-conjunctival approach to orbit. Further lateral extension of the incision is carried to the preauricular region, and then continued as a hemicoronal scalp incision (Fig. 3).

The intraoral incision includes a midline incision as continuation of the Webber-Ferguson incision between the maxillary central incisors. This is followed by incision along maxillary palatal gingivae to ipsilateral tuberocity of maxilla.

Depending on the type of facial translocation employed a limited part of the incision can be used or it can be extended to the opposite side and also to include lower lip/mandible split approach.

Exposure: Facial bone is exposed along the proposed osteotomy lines. Care should be taken to retain soft tissue over the bone to maintain its vascularity. During the exposure attention should be focussed on three key anatomic structures:

- Frontal branches of facial nerve

- Infra-orbital nerve

- Lacrimal duct

- Dental Occlusion

The Frontal branches of facial nerves have to be divided as part of the exposure in a significant number of cases. To facilitate reanastomosis during reconstruction, it should be identified along the horizontal facial incision. 
This can be aided by facial nerve monitoring. About three to five of these branches are usually present. For ease of reanastomosis these branches can be intubated with $3 \mathrm{~mm}$ silicone tubes, which is split along one of its walls. The silicone tube is then retained in place by a 6-O nylon suture at either ends. The tube is then divided at its middle. At the time of reconstruction the transected ends of silicone tubes could be reconnected and the continuity of facial nerve could be reestablished. In certain situations, it is necessary to extent the lateral facial incision only about $1.5 \mathrm{~cm}$ from the lateral canthus. This would allow preservation of the facial nerve function.

Modification to the classic technique was made avoiding incision connecting the lateral canthus and the preauricular incisions. This prevented the inevitable frontal branch division required in the classic approach.

The infra-orbital nerve also could be managed using a similar technique, if its root is not necessary to be sacrificed as part of skull base resection.
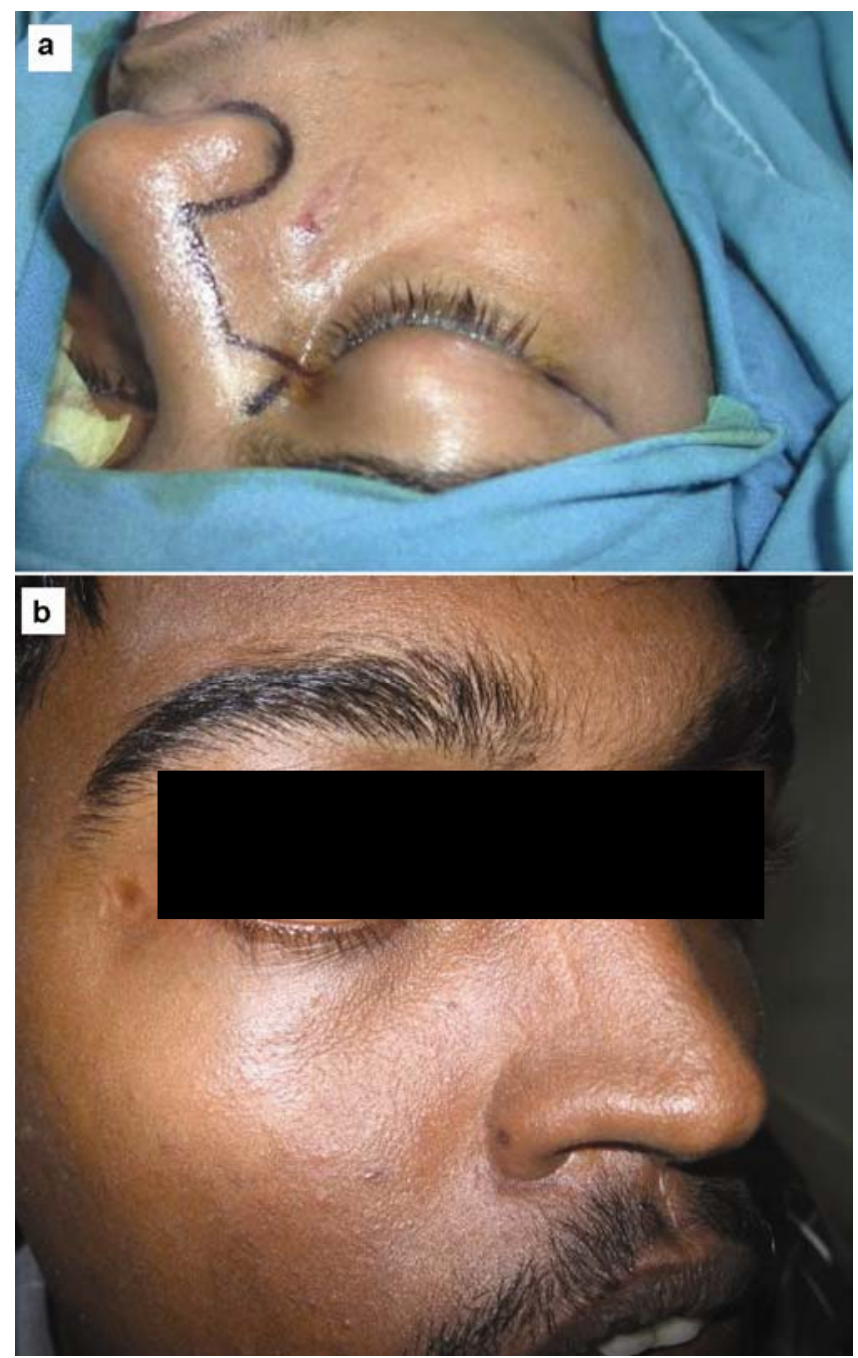

Fig. 3a Modified webber-fergusson incision to following aesthetic sub-units of face to improve aesthetic result. b Post operative result
As facial translocation involves transection of lacrimal $d u c t$, it is necessary to use a lacrimal stent at the completion of the procedure. This will need identification and dilatation of lacrimal canaliculi and intubation, which is then brought out through the naso-lacrimal duct to the nasal cavity.

The dental occlusion can be can be maintained by fabricating an acrylic dental splint preoperatively, which can be used to relocate the dentition. In addition pre-adapting the bone plates prior to osteotomy would help anatomic repositioning of the facial skeleton.

Osteotomy: Osteotomy for the 'standard facial translocation' would be described here. Either part of this or extension to contralateral side and to include mandible could be employed depending on the type of approach. (Figs. $4 \mathrm{a}$ and $\mathrm{b}$ ).

Prior to performing osteotomy bone plated should be pre-adapted along osteotomy lines. Fronto-nasal and fronto-zygomatic sutures are first separated using a fine reciprocating blade. Both these cuts are then extended for
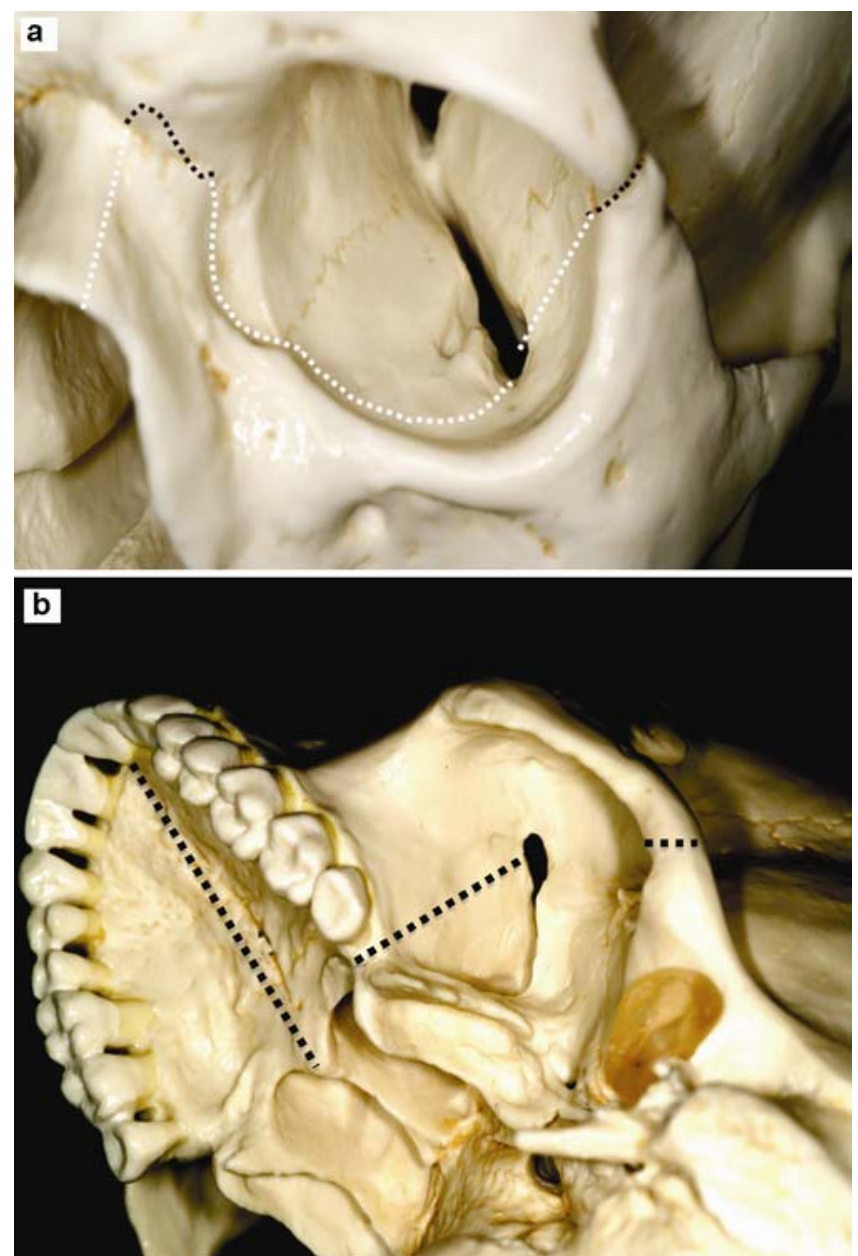

Fig 4a Osteotomy for standard facial translocation. b Osteotomy for standard facial translocation 
about $1 \mathrm{~cm}$ into medial and lateral orbital walls respectively. Using a right-angled fine saw blade orbital floor osteotomy is made to connect the medial and lateral orbital wall osteotomies. Using the same saw zygomaticotemporal suture is separated along the lateral wall of orbit to the inferior orbital fissure. Care should be taken during this procedure to protect the periorbita and the orbital contents. The frontonasal osteotomy is then extended vertically to the pyriform fossa in a paramedian plane. This is followed by division of zygomatic arch just anterior to tempero-mandibular joint. Attention can then be focussed on separating the maxilla at a paramedian position along the hard palate. Pterygo-maxillary disjunction can then be made using a large curved osteotome, which completes separation of mid-face from the skull base and adjacent structures. The mid-face can then be dislocated along with the Webber-Ferguson flap using finger pressure. This will allows opening the face like a book providing a wide exposure of the middle cranial fossa. Orbito-temporal or Pterional craniotomy can be performed for intracranial access.

Reconstruction: Dural repair is performed by conventional techniques. A galeopericranial flap may assist in this process. Significant defects of middle cranial fossa floor require either bone graft or using titanium mesh and Bone source. The orbital volume should be restored using titanium mesh or bone graft. Osteotomized facial skeleton can be repositioned and stabilized in its anatomic location using the preadapted bone plates. Skull base dead space can be obliterated and further support to orbital floor can be achieved either by using temporalis muscle flap or rectus abdominis free flap. Should osseous reconstruction of maxilla is required a deep circumflex ileac artery based ileac bone flap with internal oblique muscle may be used. Final stabilization of the dental arch can be achieved using an acrylic dental splint fabricated preoperatively. (Figs $5 \mathrm{a}$ and $\mathrm{b}$ ).

\section{Midfacial degloving intraoral incision}

A midfacial degloving approach is a well-recognized approach to the paranasal sinuses that avoids a facial incision. It is mostly combined with either a bifrontal craniotomy or a subcranial approach. Most commonly this approach is used when bilateral medial maxillectomy is planned. This is a very good approach for avoiding any facial scar and gives excellent exposure to nasal cavities.

\section{Reconstruction of skull base defects}

Ever since the first series of craniofacial resection was published in 1960's, reconstructive options have evolved continuously. Initial attempts at skull base reconstruction, which consisted of closure of dural defects with skin grafting, yielded hazardous results with complications like cerebrospinal fluid reaching up to $71 \%$. ${ }^{36}$ Subsequent techniques used local flaps like glabellar, forehead, temporalis and pericranial for reconstruction, which gave better outcome but was still not full filling all the requirements. Inadequate volume to support the skull base after complex resection was one of main limiting factor. But in large proportion of anterior skull base defects, regional flaps remain the choice of reconstruction.

In 1970's, use of myocutaneous flaps like pectoralis major, latissimus dorsi and trapezious became popular. Though bulk was not a problem with these flaps, adequate reach at distant skull base sites was difficult at times. Necrosis of distal most portion of flap and detachment from primary site due to weight dragging the flap down were major problems.

During 1980's and 1990's, microvascular techniques improved and their use in skull base reconstruction became popular. With better understanding of flap design

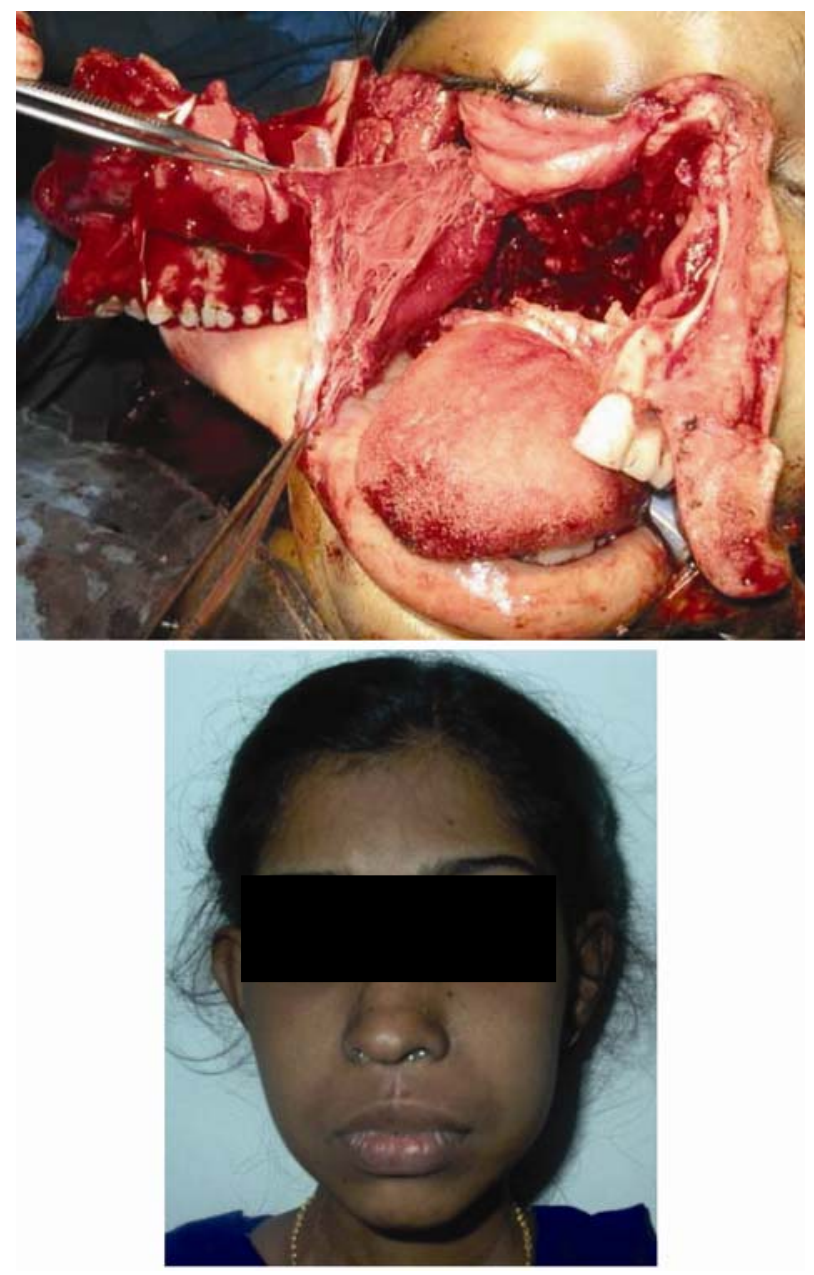

Fig. 5a Facial translocation- exposure and use of temporalis for defect reconstruction. b Facial translocation approach- post operative appearance 
and improvement in microvascular technique, results have steadily improved. ${ }^{36-49}$ Peter Neligen et al in 199643, described single institute experience of skull base reconstruction in 90 patients using local, regional or free flap. Overall complication rate in local flap and free flap group was approximately similar (38.8\% vs $33.5 \%)$. But complication rate in regional flap group was significantly higher $(75 \%)$. They concluded saying use of combination of local and free flap according to requirement of individual case probably gave best results.

\section{Anatomical and functional requirements of skull base reconstruction}

The reconstructive procedures carried out for skull base resections should aim at providing following:

- Seal of dura

- Cranio-nasal separation and support to dura

- Cover to carotid artery

- Support to orbital contents

- Oro-nasal separation

- Good cosmetic and functional outcome

CSF leak is probably the most important post-operative complication, which leads to various CNS complications and to increase morbidity and mortality. Watertight seal of dura is of utmost important. Small rents in dura can be closed primarily but multiple rents or larger defects need to be re-in forced. To achieve this, either local flaps like pericranialgalea flap and temporalis fascia flap or distant flaps like fascia lata can be used. Both are equally effective in efficiently sealing dura. Fibrin glue has also been used with these flaps to effectively seal the dura. Use of fascio-cutaneous or fascia only free flaps for dural closure has become popular recently particularly in postsurgery or post-RT redo settings.

After resection of skull base tumour, cranial cavity is often exposed to adjacent nasal or oral cavity, which serves as potent source of infection. Resulting dead space can cause hematoma and serous collection. It is advisable to separate cranial cavity from adjacent open cavities to prevent hazardous infection related complications. Local flaps like pericranial and temporal fascia flaps effectively does this in most cases. If defect is larger or posterior, then distant vascularised tissue in form of free radial forearm flap or free rectus abdominis flap is needed. These flaps provide vascularised tissue that prevent spread of infection, fill the defect and also supports the cranial base.

Bony framework is rarely required to support cranial base, unless extensive area of bone is removed. Aim of bony reconstruction is mainly to give support to cranial base and to achieve good cosmetic outcome. Removal of major part of orbital roof with cribriform plate, frontal bar or malar eminence needs bony reconstruction. Outer calvarial graft with peri-cranial flap or free bone grafts can be used. Titanium plate also serves the same function in certain cases. Free vascularised bone flaps are used in cases where adjuvant radiation is likely.

Carotid blow out due to spread of infection is a dreaded complication and to be avoided at any cost. It is essential to provide cover to carotid with vascularised tissue particularly when it is exposed in length after extensive resection. Vascularised muscle flap is an ideal flap for this purpose.

Orbital wall is sacrificed in maxillary tumour involving the orbital floor, or ethmoid tumors involving the medial wall of orbit. This can lead to increased orbital volume causing enophthalmos and diplopia. Restoration of orbital volume and support is essential for large orbital wall defect, particularly when the peri-orbita is sacrificed during resection.

Oro-nasal separation is important for functional rehabilitation of post-maxillectomy patients. Various methods ranging from use of obturator to soft tissue or bone flaps are described. Dental rehabilitation should be kept in mind in all cases while selecting reconstructive option.

Most patients under going skull base resection would receive radiation. This is an important factor in selecting reconstructive option as non-vascularised tissue or implant related complication rate is higher with radiation.

\section{Anterior skull base reconstruction}

Main focus in anterior skull base reconstruction is to achieve watertight closure of dura and provide adequate bulk and support to cranial base, which also separates cranial cavity from nasal cavity. Re-alignment of cosmetic integrity is also equally important.

Orbital floor reconstruction is essential to prevent diplopia. Oro-nasal separation enables patients to take food orally. Local-regional or free tissue can be used alone or in combination to achieve all goals.

\section{Local and regional flaps}

Small rents in dura can be repaired primarily but any defect needs reinforcement. For smaller defects, most commonly used flap is pericranial flap $^{50,51}$ or galeopericranial flap. ${ }^{52-54}$ Pericranial flap consists of periosteum and sub-aponeurotic connective tissue and is based on supratrocheal or supraorbital arteries. This flap can also be based laterally on branches of deep temporal artery. ${ }^{55}$ Galea and part of frontalis muscle is included along with pericranial flap when more bulk is required and this has a better vascularity. Contour deformity of forehead or necrosis of skin can rarely occur if galeapericranial flap is used. Previous surgery or radio- 
therapy can be a relative contraindication for use of local flaps. The flap can be passed either beneath the supraorbital rim if one-piece fronto-orbital osteotomy is used: or above the supraorbital rims in gap between frontal bone and orbital rim, if classical anterior craniofacial resection is done.

Need for multi-layer dural repair is still controversial. Larger defects at anterior skull base can pose theoretical risk of post-operative meningoencephalocele. More rigid fixation combining pericranial flap with other tissues and implants is described by some authors. ${ }^{56-58}$ Gok et al. ${ }^{56}$ published series of 17 patients with anterior skull base defects. He used pericranial flap 'sandwitched' between two layers of non-vascularised fascia lata in a threelayered repair of dura. He noticed very good results with no CSF leak, meningitis or brain herniation. Badie et al57 used titanium plate with pericranial flap for reconstruction. Incidence of CSF leak was approximately $15 \%$ in this series.

Sinha et al.$^{58}$ presented series of 20 patients with anterior skull base defects. Patients had more extensive defects combining dural defect with bony defect. Five of these patients had bilateral roof defects. He used titanium plate supported with calvarial bone grafts. These structures were draped in peri-cranial flap that was sealed at edge of dural defect with fibrin glue. After one year, no patients had CSF leak, meningitis or implant extrusion. Only 6 patients received post-operative RT in this group.

\section{Free flaps}

Choice of free flap is dictated by extent of resection done, volume and different tissue component required. Different free flaps have different tissue component like muscle, bone and skin. Choice of flap can be made based on the tissue requirement and pedicle length.

Use of radial forearm flap is suitable for defects where local tissue is not available and extensive volume or bony defects are not there. It is also ideal where skin of nose and forehead is also excised. Distinct advantage is pedicle length and pliability of tissue, can be raised as fasciocutaneous or fascia only flap. Schwartz et al reported use of radial forearm flap in 10 cases of anterior skull base. Author reports only one early CSF leak and no other complications. ${ }^{26}$ Other paper from university of Michigan, reported 20 cases of free radial forearm flaps in anterior and lateral skull base that had prior surgery or radiation. CSF leak and meningitis was reported $5 \%$ and overall complication rate was $15 \%$.

Free Antero-lateral thigh (ALT) flap can also be used in larger defects where area required to cover the defect is more but excess volume is not required. ${ }^{59,60}$ It is also very useful in a complex excision where large skin paddle is required, can be used with other flaps in combination. It has a better donor site morbidity then radial forearm flap and pedicle length can be achieved up to $20 \mathrm{~cm}$. Channa et al ${ }^{61}$ reported seven cases where free ALT flaps were used for reconstruction with no CNS complications. They also harvested fascia lata to achieve vascularised layer for dural closure.

Rectus myocutaneous flap is the most widely used free flap in anterior skull base defects. It provides large vascularised muscle that can be used to provide volume, support skull base and cover carotids. Large skin paddle can also be raised on various axis that is useful in complex defects. Rectus has a long pedicle and harvest is simple and simultaneous. It can be harvested with rib that can be used for orbital floor reconstruction. All these factors make rectus an ideal flap for large volume and complex 5 defects. Large series of 35 patients published by Teknos et al. ${ }^{62}$, included reconstruction with free rectus abdominis flap in 20 patients. CSF leak was reported at $8.6 \%$ and meningitis at $2.9 \%$.

\section{Alloplasts in skull base reconstruction}

In the past 10 years, advances in bone implant technology have yielded several new and exciting alloplastic materials with applications to skull base surgery. ${ }^{63-71}$ They include titanium plate, hydroxyapatite cement porous polyethylene and resorbable plate fixation. Implants should be used with caution as most of these patients would receive radiation and implant extrusion rates are high in these circumstances. As a rule, implants should be covered by vascularised tissue.

Titanium plates are biocompatible and can easily be adapted to fit the bony curvature. Titanium mesh is used as rigid construction for bony defect at skull base, orbital rim and frontal bone. Hydroxyapatite cement is a bonesubstitute material composed of interlinked calcium phosphate chains. It also acts as bone conductive material and is said to undergo incorporation and eventually replacement by bony in-growth. It is supplied as powder and liquid, which is to be mixed intra-operatively to yield moldable gel. Once this is molded into required shape and left in situ for setting. Porous polyethylene implants are characterized by in-growth of fibrovascular tissue and has been used for long in cranio-facial trauma cases. Resorbable plates and screws are useful for pediatric age group where growth of skeleton is not restricted by rigid plates.

External prosthesis can also be used alone or in combination with flaps to give better cosmetic outcome. Nasal prosthesis and orbital prosthesis with spectacles are used frequently.

\section{Complications}

Principal complications of skull base surgery are bleeding, CSF leak and infection. Venous bleeding can be 
Table 1. Disease outcome of anterior skull base surgery

\begin{tabular}{lccc}
\hline Reference & Patients & DFS (\%) & Adjuvant RT \\
\hline Lund $^{22}$ & 167 & 44 & DNR \\
Bridger $^{76}$ & 73 & 69 & $100 \%$ \\
Bentz $^{16}$ & 166 & 41 & $49 \%$ \\
Suarez $^{75}$ & 100 & 57 & $55 \%$ \\
Ganley $^{74}$ & 334 & 53.3 & $48.2 \%$ \\
Patel $^{77}$ & 1307 & 54 & $39 \%$ \\
\hline
\end{tabular}

Table 2. Different skull base procedures in our study

\begin{tabular}{lc}
\hline Procedure & Number of cases \\
\hline Anterior skull base & 31 \\
Antero-lateral skull base & 25 \\
Lateral-posterior skull base & 8 \\
Total & 64 \\
\hline
\end{tabular}

Table 3. Complications

\begin{tabular}{lcccc}
\hline Complication & Anterior (31) & Antero-lateral (25) & Lateral-posterior (8) & Total (64) \\
\hline Cerebrospinal fluid leak & 4 & 6 & - & 10 \\
Pneumocephalus & 4 & - & - & 4 \\
Meningitis & 3 & 3 & - & 6 \\
Wound infection & 3 & 4 & 1 & 8 \\
Chest infection & 2 & - & - & 2 \\
Bone complication & 1 & 2 & - & 3 \\
Flap loss & 2 & 2 & - & 4 \\
\hline
\end{tabular}

encountered at the skull base and from the pterygoid venous plexus. Electrocautery, bone wax and Gelform could be used for effective haemostasis. Arterial bleeding from carotid and vertebral vessels requires temporary clamping and shunting using Javid shunt and repair of the bleeding vessel. Anticipation and adequate preparation for such troublesome complication by appropriate vascular imaging and carotid occlusion study are the key to success.

CSF leak with threat of meningitis should be recognized and treated aggressively. Beta-2-transferrin electrophoresis of suspected fluid is currently the most accurate test to document CSF leak. Adequate repair of dural defects using temporalis fascia or fascia lata with fibrin glue, and fat graft are required to prevent such complication. Galeopericranial flap would further assist in this process.

The primary cause of infection is the failure to obliterate skull base dead space with vascularized tissue. Skull base infection should be treated promptly and aggressively. In the presence of unobliterated dead space, treatment with antibiotics alone would be futile. It would be necessary to bring in vascularized tissue to the site of infection. This can be either temporalis muscle flap or a rectus abdominis free flap. However the timinig is criti- cal, as most of the patients with established infection may not withstand long procedures. Wound healing problem is encountered most commonly in patients who had previous radiation therapy or surgery.

\section{Outcome}

Disease outcome of anterior skull base surgery has improved steadily over the years. In a systematic review by Dulgerov et al. ${ }^{73}$ the overall disease free survival in 1960 's was $28+/-13 \%$, where as in 1990 s it improved to $51 \pm 14 \%$. Current literature reports disease free survival between 41 to $69 \%$ (Table 1 ). The outcome depend on the extent of tumor involvement, resection status and the histopathology. Report of international collaborative study on anterior skull base tumor reports that of all the prognostic factors surgical margin status was the critical prognostic indicator. With negative margin the disease free survival was $73.5 \%$ where as with positive margin it was $37.8 \%^{77}$

Sixty four patients were operated at our institute for skull base resection. 31 had anterior skull base surgery while 24 had antero-lateral resection. Seven patients un- 
der went lateral skull base surgery and one had posterior skull base resection (Table 2). Results are shown in Table 3 . Out of 64 patients, seven died of perioperative complication. Most common complication was cerebrospinal fluid (CSF) leak. Five out of seven patients who died had received previous treatment. Four of these patients had other co-morbidities. There were multiple contributing factors like CSF leak, flap loss and wound infection and sepsis compounding the problem.

\section{Conclusion}

Surgery of craniofacial tumors is now a well-established subspecialty. Several advances such as the facial translocation approach have improved the operability and safety of these procedures. It is incumbent upon all surgeons practicing in this field to continue to strive for further improvements in these areas.

\section{References}

1. Smith RR, Klopp CT, Williams JM Surgical treatment of cancer of the frontal sinus and adjacent areas. Cancer. 1954;7:991-4.

2. Dandy WE. Orbital tumor: results following the transcranial operative attack, New York: Oskar Priest, 1941.

3. Rae BS, McLean JM. Combined intracranial and orbital operation for retinoblastoma. Arch Ophthalmol. 1943;30:437-45.

4. Ketcham AS, Wilkins RH, Van Buren $\mathrm{JM}$, et al. A combined intracranial approach to the paranasal sinuses. Am J Surg. 1963;106:698703

5. Van Buren JM, Ommaya AK, Ketcham AS. Ten years' experience with radical combined craniofacial resection of malignant tumors of the paranasal sinuses. J Neurosurg. 1968;28: 341-50.

6. Kaplan MJ, McDermott MW, Gutin $\mathrm{PH}$, et al. Transcutaneous transfacial approaches to the anterior skull base. In Operative techniques in neurosurgery (ed. Lawton M) 2000;3:53-6.

7. Har-El G. Anterior craniofacial resection without facial skin incision a review. Otolaryngol Head Neck Surg. 2004;130:780-7.

8. Lang DA. Surgery of the cranial base. In Surgery of Cranial Base Tumors (eds Sekhar LN, Janecka IP), New York, Raven Press, 1993; pp. 93-121.

9. Jho HD, Ha HG. Endoscopic endonasal skull base surgery: Part 1 the midline anterior fossa skull base. Minimum Invasive Neurosurg 2004;47:1-8.

10. Iannetti $G$, Valentini V, Rinna $C$, Ventucci E, Marianetti TM. Ethmoido-orbital tumors: our experience. J Craniofac Surg 2005; 16:10851091.
11. Tiwari $R$, van der Wal $J$, van der Waal I, et al. Studies of the anatomy and pathology of the orbit in carcinoma of the maxillary sinus and their impact on preservation of the eye in maxillectomy. Head Neck. 1998;20(3):193-6.

12. Ibrahim HZ, Moir MS, Fee WW. Nasopharyngectomy after failure of 2 courses of radiation therapy. Arch Otolaryngol Head Neck Surg. 2002;128:1196-7.

13. To EW, Yuen EH, Tsang WM, et al. The use of stereotactic navigation guidance in minimally invasive transnasal nasopharyngectomy: a comparison with the conventional open transfacial approach. Br J Radiol. 2002;75(892):345-50.

14. To EW, Lai EC, Cheng JH, et al. Nasopharyngectomy for recurrent nasopharyngeal carcinoma: a review of 31 patients and prognostic factors. Laryngoscope. 2002;112:1877-82.

15. Myers LL, Oxford LE. Di erential diagnosis and treatment options in paranasal sinus cancer. Surg Oncol Clin N Am. 2004;13:167-86.

16. Bentz BG, Bilsky MH, Shah JP, et al. Anterior skull base surgery for malignant tumors: a multivariate analysis of 27 years of experience. Head Neck. 2003;25:515-20.

17. Kaplan MJ, Fischbein NJ, Harsh GR. Anterior skull base surgery. Otolaryngol Clin N Am. 2005;38: 107-131.

18. Le QT, Fu KK, Kaplan MJ, et al. Lymph node metastasis in maxillary sinus carcinoma. Int J Radiat Oncol Biol Phys. 2000;46(3):541-9.

19. Pitman KT, Prokopakis EP, Ay$\operatorname{dog} a n \mathrm{~B}$, et al. The role of skull base surgery for the treatment of adenoid cystic carcinoma of the sinonasal tract. Head Neck. 1999;21(5):402-7.
20. Dias FL, Sa GM, Lima RA, et al. Patterns of failure and outcome in esthesioneuroblastoma. Arch Otolaryngol Head Neck Surg. 2003;129: 1186-92.

21. Bradley PJ, Jones NS, Robertson I. Diagnosis and management of esthesioneuroblastoma. Curr Opin Otolaryngol Head Neck Surg. 2003; 11:112-8.

22. Lund VJ, Howard D, Wei W, et al. Olfactory neuroblastoma: past, present, and future? Laryngoscope. 2003;113:502-7.

23. Brandwein MS, Rothstein A, Lawson $\mathrm{W}$, et al. Sinonasal melanoma. A clinicopathological study of 25 cases and literature meta-analysis. Arch Otolaryngol Head Neck Surg 1997; 123: 290-6.

24. Patel SG, Prasad ML, Escrig M, et al. Primary mucosal malignant melanoma of the head and neck. Head Neck. 2002;24:247-57.

25. Kramer D, Durham JS, Sheehan F, et al. Sinonasal undi erentiated carcinoma: case series and systematic review of the literature. J Otolaryngol. 2004;33:32-6.

26. Kim BS, Vongtama R, Juillard G. Sinonasal undi erentiated carcinoma: case series and literature review. Am J Otolaryngol. 2004;25: 162-6.

27. Chan AW, Pommier $P$, Deschler DG, et al. Combined proton radiotherapy with chemotherapy for advanced sinonasal neuroendocrine carcinoma [abstract]. Proceedings of the Sixth International Conference on Head and Neck Cancer. Washington, DC, 2004. p. 293

28. Har-El G. Anterior craniofacial resection without facial skin incisions -a review. Otolaryngol Head Neck Surg. 2004;103:780-7. 
29. Thaler ER, Kotapka M, Lanza DC, et al. Endoscopically assisted anterior cranial skull base resection of sinonasal tumors. Am J Rhinol. 1999;13:303-10.

30. Yuen APW, Fung CT, Hung KN. Endoscopic cranionasal resection of anterior skull base tumor. Am J Otolaryngol. 1997;18:431-3.

31. Har-El G, Todor R. Anterior craniofacial resection without facial skin incisions. Skull Base. 2003;13(Suppl 1):13-20.

32. Fagan JJ, Snyderman CH, Carrau RL, et al. Nasopharyngeal angiofibromas: selecting a surgical approach. Head Neck. 1997;19:391-9.

33. Carrau RL, Snyderman CH, Kassam $A B$, et al. Endoscopic and endoscopicassisted surgery for juvenile angio.broma. Laryngoscope 2001; 111:483-7

34. Al-Nashar IS, Carrau RL, Herrera A, et al. Endoscopic transnasal transpterygopalatine fossa approach to the lateral recess of the sphenoid sinus. Laryngoscope. 2004;114:52832.

35. Raveh J, Laedrach K, Speiser M, et al. The subcranial approach for frontoorbital and antero-posterior skull base tumors. Arch Otolaryngol Head Neck Surg. 1993;119:382-93.

36. Ketcham AS, Wilkins $\mathrm{RH}$, Van $\mathrm{Bu}-$ ren $\mathrm{JM}$, et al. A combined intracranial facial approach to the paranasal sinuses. Am J Surg 1963;106:698.

37. Urken ML, Catalano PJ, Sen C, et al. Free tissue transfer for skull base reconstruction analysis of complications and a classification scheme for defining skull base defects. Arch Otolaryngol Head Neck Surg. 1993; 119:1318-1325

38. Kraus DH, Shah JP, Arbit E, et al. Complications of craniofacial resection for tumors involving the anterior skull base. Head Neck. 1994;16:307-312

39. Catalano PJ, Hecht CS, Biller HF, et al. Craniofacial resection. An analysis of 73 cases. Arch Otolaryngol Head Neck Surg. 1994;120:12031208.

40. Irish JC, Gullane PJ, Gentili F, et al. Tumors of the skull base: outcome and survival analysis of 77 cases. Head Neck 1994;16:3-10. Good description of a sitebased classification scheme of skull base tumors.
41. Janecka IP, Sen C, Sekhar LN, et al. Cranial base surgery: results in 183 patients. Otolaryngol Head Neck Surg. 1994;110:539-546.

42. Clayman GL, DeMonte F, Jaffe DM, et al. Outcome and complications of extended cranial-base resection requiring microvascular free-tissue transfer. Arch Otolaryngol Head Neck Surg. 1995;121:1253-1257.

43. Neligan PC, Mulholland S, Irish J, et al. Flap selection in cranial base reconstruction. Plast Reconstr Surg. 1996, 98:1159-1166.

44. McCutcheon IE, Blacklock JB, Weber RS, et al. Anterior transcranial (craniofacial) resection of tumors of the paranasal sinuses: surgical technique and results. Neurosurgery 1996;38:471-479; discussion 479480.

45. Shah JP, Kraus DH, Bilsky MH, et al. Craniofacial resection for malignant tumors involving the anterior skull base. Arch Otolaryngol Head Neck Surg. 1997; 123:1312-1317.

46. Dias FL, Sa GM, Kligerman J, et al. Complications of anterior craniofacial resection. Head Neck. 1999; 21:12-20.

47. Solero CL, DiMeco F, Sampath P, et al. Combined anterior craniofacial resection for tumors involving the cribriform plate: early postoperative complications and technical considerations. Microsurgery 2000;47: 1296-1304; discussion 1304-1305.

48. Chang DW, Langstein HN, Gupta A, et al. Reconstructive management of cranial base defects after tumor ablation. Plast Reconstr Surg. 2001;107:1346-1355.

49. Heth JA, Funk GF, Karnell LH, et al. Free tissue transfer and local flap complications in anterior and anterolateral skull base surgery. Head Neck. 2002;24:901-911.

50. Price JC, Loury M, Carson B, et al. The peri-cranial flap for reconstruction of anterior skull base defects. Laryngoscope. 1988;98:11591164.

51. Argenta LC, Friedman RJ, Dingman $\mathrm{RO}$, et al. The versatility of pericranial flaps. Plast Reconstr Surg 1985; 76:695-702.

52. Jackson IT, Adham MN, Marsh WR: Use of galeal frontalis myofascial flap in craniofacial surgery. Plast Reconstr Surg. 1986;905-910.
53. Schramm VL, Myers MN, Maroon JC: Anterior skull base surgery for benign and malignant disease. Laryngoscope. 1979;89:1077-1091.

54. Horowitz JH, Persing JA, Nichter LS, et al. Galeal-pericranial flaps in head and neck reconstruction. Am J Surg. 1984;148:489-497.

55. Potparic Z, Fukuta K, Colen LB, et al. Galeo-pericranial flaps in the forehead: a study of blood supply and volumes. $\mathrm{Br} J$ Plast Surg. 1996;49:519-528

56. Gok A, Erkutlu I, Alptekin M, et al. Three-layer reconstruction with fascia lata and vascularized pericranium for anterior skull base defects. Acta Neurochir. 2004;146:53-57.

57. Badie B, Preston JK, Hartig GK. Use of titanium mesh for reconstruction of large anterior cranial base defects. J Neurosurg. 2000;93:711714.

58. Sinha UK, Johnson TE, Crockett D, et al. Three-layer reconstruction for large defects of the anterior skull base. Laryngoscope. 2002;112:424427.

59. Iida $H$. The advantage of the anterolateral thigh flap for reconstruction of the anterior skull base defect in recurrent cases. Plast Reconstr Surg. 2003; 112:703-704.

60. Marchetti C, Gessaroli M, Cipriani $\mathrm{R}$, et al. Use of 'perforator flaps' in skull base reconstruction after tumor resection. Plast Reconstr Surg. 2002; 110:1303-1309.

61. Chana JS, Chen HC, Sharma R, et al. Use of the free vastus lateralis flap in skull base reconstruction. Plast Reconstr Surg. 2003;111:568574. Describes the versatility of the anterolateral free flap for reconstructions of the skull base.

62. Teknos TN, Smith JC, Day TA, et al. Microvascular free tissue transfer in reconstructing skull base defects: lessons learned. Laryngoscope. 2002; 112:1871-1876

63. Janecka IP. Nelw reconstructive technologies in skull base surgery: role of titanium mesh and porous polyethylene. Arch Otolaryngol Head Neck Surg. 2000;126:396401.

64. Zide MF, Kent JN, Machado L. Hydroxylapatite cranioplasty directly over dura. J Oral Maxillofac Surg. 1987;45:481-486. 
65. Kveton JF, Friedman CD, Costantino PD. Indications for hydroxyapatite cement reconstruction in lateral skull base surgery. Am J Otol. 1995; 16:465-469.

66. Weissman JL, Snyderman $\mathrm{CH}$, Hirsch BE. Hydroxyapatite cement to repair skull base defects: radiologic appearance. AJNR Am J Neuroradiol. 1996;17:15691574.

67. Ducic Y Three-dimensional alloplastic orbital reconstruction in skull base surgery. Laryngoscope 2001; 111:1306-1312.

68. Kaptain GJ, Vincent DA, Laws Jr ER. Cranial base reconstruction after transsphenoidal surgery with bioabsorbable implants. Neurosurgery. 2001;48:232-233
69. Imola MJ, Hamlar DD, Shao W, et al. Resorbable plate fixation in pediatric craniofacial surgery. Arch Facial Plast Surg. 2001;3:79-90.

70. Imola MJ, Schramm VL. Resorbable fixation in pediatric cranial base surgery: How I do it. Laryngoscope. 2002;112:1897-1901

71. Romano JJ, Iliff NT, Manson PN. Use of Medpor porous polyethylene implants in 140 patients with facial fractures. J Craniofac Surg. 1993;4:142-147.

72. Koornneeff L. New insight in the human orbital connective tissue: result of a new anatomical approach Archives of Ophthalmology. 1977; 95(7):1269.

73. Dulgurov et al., Nasal and paranasal sinus carcinoma: are we making pro- gress? A series of 220 patients and a systematic review. Cancer. 2001; $92: 12$

74. Ganly I, Patel SN, et al. Complication of craniofacial resection for malignant tumours of skull baseReport of an international collaborative study. Head Neck. 2005; 27:445-451.

75. Saurez C, et al. Anterior Craniofacial resection: oncologic outcome and complications in series of 111 patients. Acta Otorrinolaringol Esp. 2004;55(1):27-33

76. Bridger $\mathrm{PG}$, et al. Craniofacial resection for paranasal sinus cancer. Head Neck. 2000;22(8):772-80.

77. Paten $\mathrm{SN}$, et al. Craniofacial surgery for malignant skull base tumors. Cancer. 2003;98:1179-87. 